\title{
A INFLUÊNCIA DO TREINAMENTO DE FORÇA E DO TREINAMENTO AERÓBIO SOBRE AS CONCENTRAC̄ÕES HORMONAIS DE TESTOSTERONA E CORTISOL
}

\author{
THE INFLUENCE OF THE TRAINING OF RESISTANCE AND THE AEROBIC TRAINING \\ ON THE HORMONAL CONCENTRATIONS OF TESTOSTERONE AND CORTISOL
}

\author{
AUTOR \\ Marcelo Rangel de Araújo \\ ${ }^{1}$ Universidade Estadual de Londrina
}

\section{A INFLUÊNCIA DO TREINAMENTO DE FORCAA E DO TREINAMENTO AERÓBIO SOBRE AS CONCENTRAC̄ÕES HORMONAIS DE TESTOSTERONA E CORTISOL 4(2): $67-75$}

\section{PALAVRAS-CHAVE}

treinamento; testosterona; cortisol.

\section{KEYWORDS}

training; testosterone; cortisol.
RESUMO

A intenção desse trabalho é rever a influência do treinamento aeróbio e de força, perante as concentrações agudas e crônicas dos hormônios testosterona e cortisol. Além disso, verificar outros fatores importantes, tais como idade, alimentação e ritmo circadiano, os quais podem modificar agudamente as concentrações de testosterona e cortisol, dificultando assim o entendimento das respostas hormonais desencadeadas pelo treinamento. Apesar das diversas controvérsias encontradas no presente estudo, as atividades periodizadas e de alta intensidade parecem estimular maiores liberações de testosterona e pouca liberação de cortisol, potencializando, conseqüentemente, os níveis de força e hipertrofia muscular. Todavia, novos estudos devem ser realizados com delineamentos mais adequados respeitando as diversas interações (alimentação, idade, sexo, nível de experiência com o treinamento físico, etc.] que envolvem o treinamento físico.

\section{ABSTRACT}

The goal of this paper is to review the influence of aerobic training and resistance in the acute and chronic concentrations of hormones testosterone and cortisol. In addition, it looks at other important factors, such as age, feeding and circadian rhythm which can modify the concentration of testosterone and cortisol acutely thus making it difficult the understanding of the hormonal answers unchained by the training. Although the diverse controversies found in the present study, the periodical and high intensity activities seem to stimulate bigger releases of testosterone and little release of cortisol, contributing, consequently, to the levels of force and muscular hypertrophy. However, new studies need to be carried out with adjusted delineations respecting the diverse interactions (feeding, age, sex, level of experience with the physical training, etc.) that involve the physical training. 


\section{INTRODUĈ̣̃O}

\section{Biossíntese e regulação da testosterona}

A Testosterona é o principal hormônio sexual masculino. Quando suas concentrações circulantes estão baixas no organismo, o hipotálamo promove a liberação do fator liberador da gonadotropina (GnRF). O GnRF estimula a liberação do hormônio luteinizante (LH), que por sua vez, estimula as células de Leydig nos testículos a produzir e liberar testosterona $a^{12}$. Uma pequena quantidade de testosterona é secretada também pelas glândulas supra-renais. A concentração plasmática de testosterona varia de $\mathbf{3 0 0}$ a $1.000 \mathrm{ng} / \mathrm{dl}$ e a taxa de produção diária de 2,5 a $11 \mathrm{mg}^{49}$. Nas mulheres esse hormônio também é produzido pelas glândulas supra-renais e ovários, porém em menor quantidades 0,25 a $1 \mathrm{mg} / \mathrm{dia}^{45}$.

Suas funções são basicamente duas, denominadas anabólicas e androgênica. Pela função anabólica ele atua principalmente sobre as zonas de crescimento dos ossos e músculos, além de influenciar o desenvolvimento de praticamente todos os órgãos do corpo humano. Pelo lado androgênico, ele é responsável pelo desenvolvimento das características sexuais masculinas [órgãos sexuais, produção de espermatozóide, barba, etc) ${ }^{45}$.

\section{Biossíntese e regulação do cortisol}

0 cortisol é o hormônio mais importante dos chamados glicocorticóides, ele é secretado a partir de um estímulo estressante (atividade física ou contusão em alguma parte do corpol que transmite impulsos nervosos ao hipotálamo o qual libera o fator liberador de corticotropina (FLC) que chega a hipófise anterior onde suas células secretam hormônio adrenocorticotrópico que flui pelo sangue até o córtex supra-renal onde será produzido 0 cortisol $^{13}$.

O hormônio cortisol é conhecido pela sua função catabólica, exercendo um papel importante no equilíbrio eletrolítico e no metabolismo de carboidratos, proteínas e lipídeos, alem de possuir um potente efeito antiinflamatório ${ }^{13}$.

\section{Relações hormonais perante a Idade}

A testosterona parece não estar sujeita a sofrer mudanças em sua concentração basal, até aproximadamente os 10 anos de idade, onde sua concentração encontra-se por volta de 0,3 (nmol/L), Já no começo da puberdade, próximo aos 13 anos de idade, a testosterona tem um aumento significativo chegando a valores médios de 3,16 (nmol/L), e na puberdade propriamente dita, entre 13 e 14 anos a concentração de testosterona alcança valores médios de $12(\mathrm{nmol} / \mathrm{L})^{11}$. Aos 14 anos de idade os garotos apresentam valores de cortisol e testosterona igual a homens adultos saudáveis ${ }^{46}$. Com o envelhecimento, ocorre uma diminuição nas concentrações isoladas de testosterona livre e total. Após os $\mathbf{5 0}$ anos, a concentração sérica de testosterona apresenta diminuição de $1 \%$ ao ano ${ }^{14}$. Essa redução está diretamente relacionada ao hipogonadismo ${ }^{21}$. Por outro lado os níveis de cortisol tende a aumentar com o envelhecimento de mulheres, mas não necessariamente de homens, aumentando também o catabolismo muscular ${ }^{16}$.

\section{A influência da alimentação sobre as concentrações hormonais}

Um outro fator que pode modificar agudamente os níveis hormonais é a alimentação, foi o que constatou o estudo de (Kraemer, et. al. 1998) o qual suplementou um grupo com carboidratos e proteínas duas horas antes e imediatamente após o exercício, favorece a redução dos níveis de cortisol e testosterona sanguíneo, após aproximadamente 15 minutos ao final do treinamento. Por outro lado os níveis de insulina aumentam consideravelmente após este período. Da mesma forma Bloomer et. al. (2000) ${ }^{1}$ verificou esta mesma relação da insulina com a testosterona, quando os grupos ingeriram uma refeição completa (carboidrato, gordura e proteína) ou somente uma bebida rica em carboidratos, ou somente uma suplementação constituída de proteína e carboidrato, imediatamente, 2 e 4 horas após a sessão de treinamento. 0 grupo que não ingeriu nenhum tipo de alimento manteve os níveis de testosterona altos, proporcionando uma ótima relação testosterona/cortisol.

Apesar do pequeno número de estudos revisado, esses dois hormônios (insulina e testosterona) parecem agir inversamente, pois quando um hormônio está em pico o outro está em baixa concentração na corrente sanguínea. Portanto, nestas condições, a presença da insulina parece ter maior importância para o anabolismo muscular, pois, assim como a testosterona à insulina também é um hormônio anabólico e, além disso, a síntese muscular só ocorre perante a presença de açucares e principalmente de proteínas, sendo assim fundamental a ingestão de proteínas e carboidratos imediatamente após o treinamento.

Uma estratégia bastante interessante para controlar os níveis de cortisol em baixas concentrações durante o estado de repouso por 
até 24 horas após um treinamento de força, parece ser a suplementação de acido ascórbico (1000mg) antes do treinamento ${ }^{36}$. Conseqüentemente proporcionando uma diminuição do catabolismo protéico em repouso.

\section{Relações hormonais perante o exercício físico e o ritmo circadiano}

As concentrações sanguíneas de testosterona podem sofrer grandes alterações durante as horas do dia, como mostra a maioria dos estudos revisados por (Loebel and Kraemer, 1998) ${ }^{34}$.

As concentrações sanguíneas de testosterona têm seu pico por volta de 6:00h as 8:00h da manhã e sofre um declínio de até 35\% durante o dia, antes de começar a aumentar novamente pelo meio da noite $^{23}$. Porém um treinamento de força intenso, realizado pelo final da tarde parece diminuir os níveis de (LH) em até $24 \%$ durante o período da noite, conseqüentemente diminuindo a produção de testosterona livre e total durante esse período $^{38}$.

Assim como a testosterona, o cortisol também parece se alterar durante as horas do dia, apresentando seu pico pelas primeiras horas da manhã. Logo ao despertar seus níveis vão declinando progressivamente ao longo do dia, ficando bastante baixos durante a noite ${ }^{30}$.

Um estudo realizado com sessões de treinamento de força, pelo período da manhã, demonstrou queda significativa na concentração dos níveis de testosterona após os exercícios, mas quando os mesmos atletas realizaram o mesmo treinamento no período da tarde, as concentrações nos níveis de testosterona aumentaram significativamente ${ }^{25}$.
Por outro lado, as menores concentrações alcançadas de cortisol, após uma sessão de exercícios de força, foram por volta das 17:00 horas, comparado com outros dois horários distintos de treinamento [7:00 e 24:00horas] ${ }^{28}$.

Desta forma pressupõe-se que o melhor horário para o treinamento de força seja pelo final da tarde e inicio da noite, onde os níveis de cortisol aumentam em menor grau e a testosterona em maior grau, proporcionando um bom estado para o anabolismo muscular.

Porém, independentemente do ritmo circadiano dos hormônios testosterona e cortisol, [Souissi et. al. $2002)^{48}$ destaca em seu estudo que os melhores resultados encontrados para a potência anaeróbia e picos de força máxima estão diretamente relacionados ao horário de treino com o horário de avaliação [testes] da capacidade física treinada. Se o treinamento é feito no período da manhã os resultados das avaliações (testes] serão melhores apresentados no período da manhã, quando comparados com avaliações realizadas pelo período da tarde, e vice-versa. Por esse motivo os atletas ou preparadores físicos devem planejar o treinamento de acordo com o horário de competição.

\section{Repostas hormonais agudas ao exercício aeróbio}

As respostas hormonais imediatamente após os exercícios aeróbicos podem variar de acordo com o grau de treinamento dos indivíduos, da intensidade, e principalmente da duração do exercício. (Jürimäe et. al. $2001]^{29}$ não verificaram mudanças significativas nos níveis de testosterona e cortisol em remadores profissionais, após remarem, a 77\% do limiar anaeróbio por aproximadamente 2 horas, certamente um estímulo de baixa intensidade não é suficiente para promover mudanças agudas no sistema endócrino em atletas. Por outro lado, corredores de elite acostumados a correr $70 \mathrm{~km}$ por semana mostraram significativas reduções nos níveis de testosterona após um teste aeróbio progressivo até o limiar anaeróbio, quando comparado a indivíduos não treinados submetidos ao mesmo teste ${ }^{6}$. Entretanto, os níveis de cortisol tendem a sofrer maiores aumentos em homens não treinados quando comparados a corredores. Além disso, a dissipação do cortisol ocorre mais lentamente nos indivíduos não treinados, após o exercício ${ }^{47}$.

Segundo (Jacks et. al. 2002) ${ }^{28}$ os níveis de cortisol, verificado através da saliva só aumentam significativamente após 59 minutos de atividade aeróbia em bicicleta ergométrica, apenas com intensidades altas (76\% do pico de V02).

Em indivíduos não treinados a testosterona pode sofrer aumentos significativos com apenas 15 a 20 minutos de exercício aeróbio moderado $^{37}$. Assim como os não treinados, homens previamente treinados acostumados a correr $16 \mathrm{~km}$ por semana, tendem a sofrer aumentos significativos nos níveis de testosterona imediatamente após $30 \mathrm{mi}-$ nutos de corrida a $80 \%$ do V02máx ${ }^{26}$. Da mesma forma (Consitt et. al. $2001]^{5}$ verificaram, em mulheres previamente treinadas aumentos significativos de testosterona, mas não de cortisol, após $\mathbf{4 0}$ minutos de corrida a $75 \%$ da freqüência cardíaca máxima, porém não houve mudanças significativas de testosterona e cortisol quando as mesmas foram submetidas a treinamento de força. 
Apesar dos diferentes resultados encontrados, certamente pelos diferentes protocolos utilizados em cada um dos estudos, os aumentos tanto de testosterona quanto de cortisol parecem ser mais evidentes em indivíduos não treinados ou previamente treinados, pois o sistema endócrino parece ser mais sensível à percepção de esforço destes indivíduos, principalmente quando o esforço tende a ser intenso.

\section{Respostas hormonais crônicas ao exercício aeróbio}

Chatard, et. al. (2002) ${ }^{4}$ analisou as concentrações basais de cortisol e DHEA antes de cada uma das 68 competições de uma "temporada", durante 37 semanas de treinamento de natação, constatando um aumento nos níveis de cortisol, mas não de DHEA conforme progredia o volume de treinamento, porém os níveis de cortisol não declinaram na fase de baixo volume (polimento). Para manter os níveis basais de cortisol reduzido durante uma temporada de natação (Filho, et. al. 2002) ${ }^{7}$ utilizou uma técnica de relaxamento progressivo, duas vezes na semana em sessões de 20 a 30 minutos, os resultados foram bem significativos quando comparado ao grupo controle.

Segundo (McArdle et. al., 1998) (77 $^{37}$ atletas profissionais que correm em média $64 \mathrm{~km}$ semanais apresentam reduzidos níveis de testosterona em repouso, quando comparados a homens não corredores da mesma faixa etária. Por outro lado, comparações feitas com corredores de altíssimo volume semanal $(94 \mathrm{~km})$, alto volume semanal [80km] e homens não acostumados a correrem, todos com a mesma faixa etária, não demonstraram diferenças significativas nos níveis de testosterona total e testosterona livre em repouso ${ }^{39}$. Da mesma forma Kraemer et. al. (1995) ${ }^{31}$ não encontraram mudanças significativas na concentração de testosterona após 12 semanas de treinamento aeróbio, mas a concentração de cortisol aumentou significativamente após a quarta semana, declinando após a oitava semana e voltando a aumentar após a décima segunda semana. Porém, um estímulo de alta intensidade e alto volume parece reduzir os níveis basais de testosterona e cortisol. É o que demonstrou o estudo de (Garcia et. al. 2002) ${ }^{10}$ realizado após 3 semanas de competição de ciclismo onde foi percorrido um total de $3781 \mathrm{~km}$.

Embora existam controvérsias entre os estudos, o que parece evidente é que os niveis basais de testosterona não tendem a aumentar em repouso com o treinamento aeróbio em longo prazo. Já os níveis basais de cortisol tendem a oscilarem mais em respostas agudas ao treinamento aeróbio, ora estando em altas concentrações ora em baixas concentrações, evidenciando assim a importância da periodização do treinamento.

\section{Respostas hormonais agudas ao treinamento de força}

Uma única sessão de exercícios de força tem demonstrado significativos aumentos na concentração de testosterona e cortisol após uma sessão de treinamento para homens $^{23,15,32,35,46}$ e mulheres ${ }^{23,43}$.

Segundo (Hansen et. al. 2001) os $^{22}$ aumentos agudos de testosterona proporcionados pelo treinamento de força, apresentam fortes correlações com o aumento da força isométrica, mas não da força máxima. Os niveis de testosterona parecem ser potencializados com métodos de cargas máximas (90 a $100 \%$ de 1RM), envolvendo grandes grupamentos musculares e longos períodos de descanso (3min) entre as séries $^{24,3,34}$. Assim como a testosterona, os níveis de cortisol são potencializados com cargas sub-máximas (60\% a75\% de 1RM) e períodos curtos de descansos

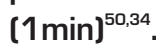

O número de séries, assim como o número de repetições empregado dentro de uma sessão de treinamento, parece exercer maior influência sobre as concentrações sanguíneas de cortisol a de testosterona, pois quanto maior o número de séries e repetições, maiores quantidades de cortisol será produzido pelo organismo sendo que os níveis de testosterona pouco se alteram em relação ao numero de séries e repeticões ${ }^{50}$ Da mesma forma (Ostrowsk et. al, 1997) ${ }^{44}$ relatam que um grande número de séries (12 séries: 4 séries de supino reto, 4 séries de supino declinado e 4 séries de supino inclinado] para o mesmo grupo muscular, dentro de uma mesma sessão de treinamento, pode proporcionar uma troca na relação testosterona/cortisol. Por outro lado apenas 1 série por grupamento muscular não é tão eficiente quanto 3 séries, para estimular o aumento da relação testosterona/cortisol, agudamente ${ }^{15}$. Um treinamento de volume balanceado com alta intensidade parece ser a melhor estratégia para potencializar os níveis de testosterona e possivelmente diminuir os níveis de cortisol, imediatamente após o exercício. Porém (Fry et. al. 2000) ${ }^{9}$ não descarta a importância do alto volume de treinamento no inicio da preparação, tanto para atletas iniciantes como para atletas experientes. 
Em contradição, existem estudos na literatura no qual o treinamento de força não proporcionou mudanças na concentração de testosterona após a sessão de treino tanto em homens 3,42 como em mulheres ${ }^{53,34}$.

Os resultados parecem ser conflitantes, pois os estudos apresentam diferentes metodologias principalmente envolvendo diferentes intensidades, que na maioria dos estudos não são máximas, possivelmente não proporcionando mudanças significativas nos níveis hormonais. Além disso, deve ser levada em consideração a variação fisiológica relativa a dieta, ritmos biológicos, estresse, doenças não endócrinas, problemas de coleta de amostras e interferências metodológicas que podem ser de várias origens e incluem anticorpos heterófilos, anticorpos endógenos anti-hormonais ${ }^{54}$.

\section{Respostas hormonais crônicas ao treinamento de força}

Pesquisas vêem demonstrando que os níveis basais de testosterona e cortisol parecem não se alterar em homens jovens com pouca experiência com treinamento de força ${ }^{23,38,33,22,20}$ homens idosos ${ }^{48}$ e mulheres idosas ${ }^{17,18}$. Por outro lado alguns estudos têm demonstrado um fator favorável para 0 anabolismo muscular mesmo sem ocorrer aumentos nos niveis basais de testosterona, através da queda nos níveis basais de cortisol sanguíneo em homens jovens ${ }^{31}$ idosos $^{27,35}$ e mulheres jovens ${ }^{53}$, após a aderência ao treinamento de força de no mínimo 8 semanas.

Embora (Kraemer et. al. 1995) ${ }^{31}$ não tenham encontrado mudanças nos níveis basais de testosterona com um treinamento de força, ocorreram acréscimos significativos de testosterona com treinamento aeróbio associado com de

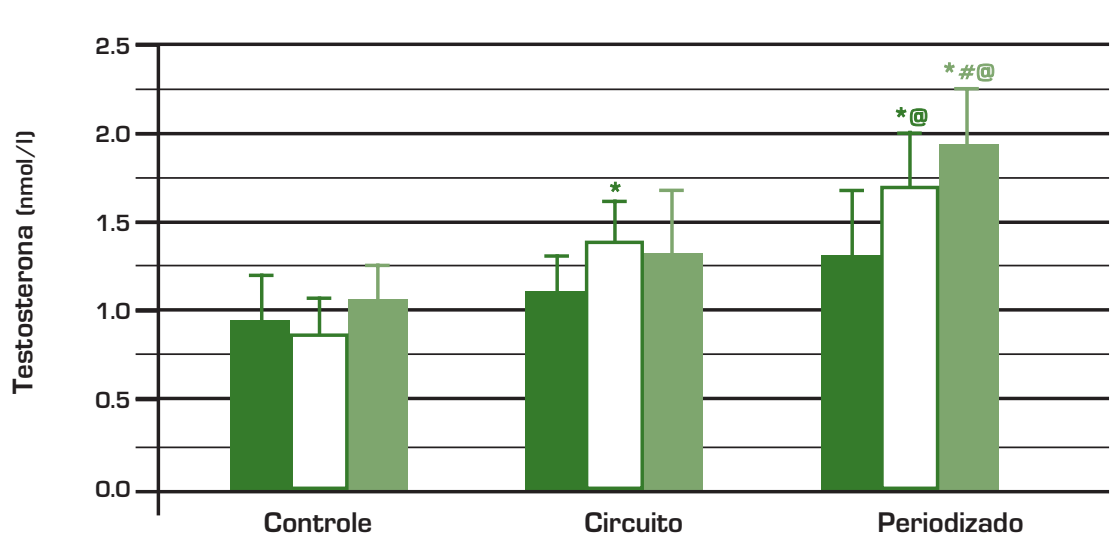

LEGENDA

Pré-treino 12 semanas

24 semanas

FIGURA 1

Concentrações basais de testosterona durante 24 semanas de treinamento de força. Efeitos do treinamento circuito e periodizado, nas concentrações basais de testosterona em mulheres jovens.

* $\mathrm{P} \leq 0,05$ corresponde ao pré-treino; \# $\mathrm{P} \leq 0,05$ corresponde a 12 semanas; $@ \mathrm{P} \leq 0,05$ corresponde à diferença entre os grupos. Adaptado de (Marx, et.al. 2001) ${ }^{41}$

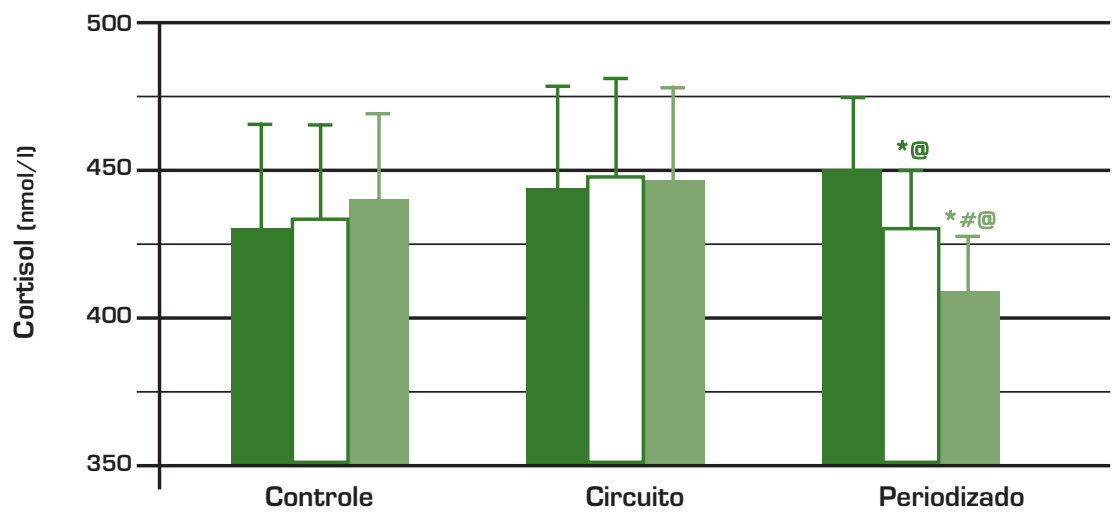

LEGENDA

Pré-treino $\square 12$ semanas $\quad 24$ semanas

FIGURA2

Concentrações basais de cortisol durante 24 semanas de treinamento de força. Efeitos do treinamento circuito e periodizado, nas concentrações basais de cortisol em mulheres jovens.

${ }^{*} \mathrm{P} \leq 0,05$ corresponde ao pré-treino; $\# \mathrm{P} \leq 0,05$ corresponde a 12 semanas; $@ \mathrm{P} \leq 0,05$ corresponde à diferença entre os grupos. Adaptado de (Marx, et.al. 2001) ${ }^{41}$ 
força após 12 semanas, mas negativamente o cortisol também teve um grande acréscimo.

Porém, (Kraemer et. al. 1999) ${ }^{35}$ verificaram que 10 semanas de treinamento de força parece proporcionar aumentos basais significativos de testosterona livre para homens de meia idade, com pouca experiência em treinamento de força, havendo também um pequeno decréscimo de cortisol. Da mesma forma (Marx et. al. 2001) ${ }^{41}$ encontraram aumentos significativos de testosterona após 12 semanas de treinamento de força, usando um programa em circuito de baixo volume e um outro periodizado de alto volume, sendo que os níveis de testosterona continuaram a aumentar por mais 12 semanas, apenas para o grupo periodizado (figura 1), sendo que no grupo circuito os níveis de cortisol não se alteraram em nenhum momento e no grupo periodizado teve um decréscimo progressivo de cortisol durante as 24 semanas (figura 2), proporcionando uma ótima relação testosterona/cortisol.

Tsolakis et. al. (2000) ${ }^{52}$ também encontraram aumentos basais significativos nas concentrações sanguíneos de testosterona em garotos de 11 a 14 anos de idade após 2 meses de treinamento. $\mathrm{E}$ surpreendentemente os niveis de testosterona alcançados não diminuíram com mais 2 meses de destreino, quando comparado ao grupo controle.

Segundo (Fry et. al. 1998) ${ }^{8}$ duas semanas de treinamento de alta intensidade $(100 \%$ de $1 \mathrm{RM})$ realizado por 6 dias na semana não alteram os niveis basais de testosterona proporcionando aumentos apenas imediatamente após o treino, mas esse tipo de treinamento proporcionou uma que de $11 \%$ da força máxima caracterizando over- training. Por este motivo (Bompa, 2001] ${ }^{2}$ limita o treinamento de força máxima em 3 sessões semanais, por no máximo nove semanas consecutivas e com poucas sessões diárias de treinamento de força máxima, admitindo desta forma significativos aumentos na produção natural de testosterona, conseqüentemente aumentando os níveis de força. É o que demonstra também o estudo feito por (Fry, et. al. 2000) ${ }^{9}$ com levantadores de peso de elite e amadores. Inicialmente os atletas realizavam de 3 a 4 sessões por dia de treinamento de força máxima, vindo a realizar posteriormente 1 a 2 sessões por dia, aumentando significativamente os níveis de testosterona em relação ao cortisol, e além disso, os atletas de elite tiveram uma grande relação com desempenho na competição após reduzirem o volume de treinamento. A periodização do treinamento de força parece ser fundamental na modulação dos níveis hormonais de testosterona e cortisol, e conseqüentemente, na potencialização da força muscular.

\section{CONSIDERAC̣ÕES FINAIS}

As mudanças nos níveis de testosterona e cortisol induzidas pelo treinamento aeróbio e treinamento de força ainda não estão bem esclarecidas. Pois as diversas interações (hora do dia, alimentação, tipo de exercício, estado de treinamento do individuo, idade, estado emocional, sexo, etc] que envolve o treinamento físico, dificultam o entendimento das respostas hormonais perante o exercício físico. De qualquer modo, a testosterona parece aumentar após sessões curtas e intensas de treinamento, principalmente de força, assim como o cortisol parece aumentar com sessões longas e intensas de treinamento, principalmente aeróbio. Além disso, programas periodizados de treinamento de força parece ser a melhor estratégia para aumentar os niveis basais de testosterona e diminuir os níveis basais de cortisol. Proporcionando assim, um estado anabólico favorável em repouso. Em todo caso novos estudos devem ser realizados, considerando principalmente populações idosas, que dificilmente sofrem alterações hormonais perante o exercício físico e o aumento da massa muscular raramente é significativo.

\section{AGRADECIMENTOS}

A minha família pelo incentivo aos estudos.

\section{CORRESPONDÊNCIA}

Marcelo Rangel de Araújo

Rua: Paulo Portezan, $n .^{\circ} 59$

Bairro: Jardim Ipê

Cep: 18900-000

Santa Cruz do Rio Pardo/

/SP/Brasil

E-mail: marcelora@pop.com.br

\section{REFERÊNCIAS}

1. Bloomer RJ, Sforzo GA and Keller BA (2000) Effects of Meal Form and Composition on Plasma Testosterone, Cortisol and Insulin Following Resistance Exercise. International Journal of Sport Nutrition and Exercise Metabolism. 10, 415-424. 
2. Bompa TO, (2001). A Periodização no Treinamento Esportivo. 1 a edição, editora Manole.

3. Bosco C, R Colli R Bonomi S, P Von Duvillard, and A Viru (2000) Monitoring Strength Training: Neuromuscular and Hormonal Profile. Med. Sci. Sport Exercise, v32, n1, 202-208.

4. Chatard JC, Atlaouil D, Lac G, Duclos M Hooper S, Mackinnon L (2002) Cortisol, Dhea, Performance and Training in Elite Swimmers. Journal Sports Med., 510-515.

5. Cositt LA, Copeland JL and Tremblay MS (2001). Hormone Responses to Resistance vs endurance Exercise in Pre menopausal Females. Can. J. Appl. Physiol., 26[6], 574-587.

6. Di Luigi L, Guidettil L, Baldaril C, Fabbri A, Moretti C, Romanelli F [2002]. Physical Stress and Qualitative Gonadotropin Secretion: LH Biological Activity at Rest and After Exercise in Trained and Untrained Men. Journal Sports Med., 23: :307-312.

7. Filho MGB, Ribeiro CS, Miranda R e Teixeira MT (2002). A Redução nos Níveis de cortisol sanguíneo através da técnica progressiva em nadadores. Revista Brasileira de Medicina do Esporte, V8, (4), 01-05.

8. Fry A C, Kraemer WJ and Ramsey LT (1998). Pituitary Adrenal-gonadal Responses to High-Intensity Resistance Exercise Over-Training. J. Appl. Phyiol., 85(6): 2352-2359.

9. Fry A C, Kraemer WJ, Stone MH, Koziris LP (2000). Thrush JT, Fleck SJ. Relationships Between Serum Testosterone, Cortisol, and Weightlifting Performance Journal of Strength and Conditioning Research, 14(3), 338-343.
10. Garcia BF, Lucía A, Hoyos J, Chicharro JL, Alonso MR, Bandres F, Terrados N (2002). The Response of Sexual and Stress Hormones of male Pro-cyclists during continuos intense competition. Journal Sports Med., 23, 555-560.

11. Garcia-Mayor RV, Andrade MA, Rios M, Large M, Dieguez C, and Casanueva FF (1997). Serum Leptin Levels in Normal Children: Relationship to Age, Gender, Body Mass Index, Pituitary-Gonadal Hormones, and Pubertal Stage*. Journal of Clinical Endocrinology and Metabolism, v.82 (9) 2849-2855.

12. Garrett WE Jr. Kirkendall DT (2003). A ciência do exercício e dos esportes. 1 a edição, Porto Alegre, editora Atrmed.

13. Gayton AC (1998). Fisiologia Humana, Gaedição. Rio de Janeiro, editora Ganabara Koogan.

14. Gebara OCE, Vieira NW, Meyer JW, Calich AG, Tai EJ, Pierri H (2002). Wajngarten M, Aldrighi JM. Efeitos Cardiovasculares da Testosterona. Arq Bras Cardiol, v.79 ( $\mathrm{n} \circ 6$ ), 644-9.

15. Gotshalk LA, Loebel CC, Nind BC, Putukian M, Sebastianelli WJ, Newton RU, Hakkinem K, Kraemer WJ (1997) Hormonal Responses of Multi-set Heavy-Resistence Exercise Protocols. Can. J. Appl. Physical., 22 (3): 244-255.

16. Gusenoff JA, Harman SM, Veldhuis JD, Jayme JJ, Clair CSt., Munzer T, Christmas C, O'Connor KG, Stevens TE, Michele F, Bellantoni MF, Pabst K, and Blackman MR (2001). Cortisol and GH secretory dynamics, and their interrelationships, in healthy aged women and men. Am J Physiol Endocrinol Metab. 280. 616-625.

17. Häkkinen K Kraemer WJ, Pakarinen A, McBride TT, McBride JM, Häkkinen A, Alen M, McGuigan MR,
Bronks R, and Newton RU [2002) Effects of resistence/power training on maximal strength, muscle morphology, and hormonal response patterns in 60-75-year-old men and women. Can. J. Appl. Physiol. 27(3), 213-231.

18. Häkkinen $K$, Pakarinen A, Kraemer WJ, Häkkinen A, Valkeinen $\mathrm{H}$, and Alen M (2001). Selective muscle hypertrophy, changes in EMG and force, and serum hormones during strength training in older women. Can. J. Appl. Physiol 91: 569-580.

19. Hanson S, Kvorning T, Kjaer M, Sjogaard G (2001). The Effect of Short-Term Strength Training on human Skeletal Muscle: The Importance of Phyfiologically elevated Hormone levels.Scand. J Med Sci Sports, 11: 347-354.

20. Haber MP, Fray AC, Rubin RM, Smith JC, Weiss WL (2004). Skeletal Muscle and Hormonal Adaptations to Circuit Weight Training in Untrained Men. J. Med. Sci. 14: :176-185.

21. Harman MT, Metter JE, Tobin JD, Pearson J, and Blackman MR (2001). Longitudinal Effects of Aging on Serum Total and Free Testosterone Levels in Healthy Men. The Journal of Clinical Endocrinology \& Metabolism. v.86, (2), 724-731.

22. Hansen S, Kuorning T, Kjaer M, Sjogaard G, (2001). The effect of short-term strength training on human skeletal muscle the importance of physiologically elevated hormone levels. J. Med. Sci. Sports, 11:347-354.

23. Hickson RC, Hidaka K, Foster C, Falduto MT and Chatterton RJJr. (1994). Successive Time Courses of Strength Development to Heavy Resistece Training. Journal Appl. Physiol. 76 (2), 663-670. 
24. Hoffman JR, Im J, Rundell KW, Kang J, Nioka S, Speiring BA, Kimer R, and Chance B (2003). Effect of Muscle Oxygenation during Resistance Exercise on Anabolic Hormone Response. Med. Sci. Sports Exerc. V. 35, No. 11, pp. 1929-1934. 25. Hoffman JR (1992). Testosterona: A Review of Fhysiological Effects and Exercise Respose. National strength end Cotidioning Association Journal. V14, n4.

26. Hughet RJ, Johnson GO, Housh TJ, Weir JP, and Kinder JE (1996). The effect of sub maximal Treadmill Running on Serum Testosterone Levels. 10 (4), 224-227.

27. Izquierdo M, Hakkinen $K$, Ibáñez J, Antón A, Garrués M, Ruesta M, and EM Gorostiaga EM (2003). Effects of strength training on sub maximal and maximal endurance performance capacity in middle-aged and older men. Journal of Strength and Conditioning $\mathrm{Re}$ search, 17(1), 129-139.

28. Jacks DE, Sowash J, Anning J, McGloughlin T, and Andres F (2002). Effect of exercise at three exercise intensities on salivary cortisol. J. Strength Cond. Res. 16 (2):286-289.

29. Jürimäe J, Jürimäe T, and Purge P [2001). Plasma Testosterone and Cortisol Response to Prolonged Sculling in Male Competitive Rowers. Journal of Sport Sciences, 19, 893-898.

30. Kanaley JA, Weltman JY, Pieper KS, Weltman A, and Hartman M (2001). Cortisol and Growth Hormone Responses to Exercise at Different Times of Day*. The Journal of Clinical Endocrinology \& Metabolism. v86 [6] 2881-2889.

31. Kraemer WJ, Patton JF, Gordon SE, Harman EA, Deschenes MR, Reynolds K, Newton RU, Triplett NT, and Dziados JE (1995). Compatibility of High Intensity end Endurence Training on Hormonal and
Skeletal Muscle Adaptations. Journal Appl. Physiol, 78(3):976-989.

32. Kraemer WJ, Volek JS, Bush JA, Putukian M, and Sebastianelli WJ (1998). Hormonal responses to consecutive days of heavy-resistance exercise with or without nutritional supplementation. J. Appl. Physiol. 85(4): 1544-1555.

33. Kraemer WJ, Koziris LP, Ratamess NA, Hakkinen K, McBride NTT, Fry AC, Gordon SE, Volek JS, French DN, Martyn R, Rubin MR, Ana L Gomez AL, Sharman MJ, Lynch JM, Izquierdo M, Newton RU, and Fleck SJ (2002). Detraining produce Minimal Changes in Physical Performance and Hormonal Variables in Recreationally Strength Trained Men. Journal of Strength and Conditioning Research, 16(3), 373-382.

34. Kraemer WJ, Fleck SJ, Dziados JE, Harman EA, Marchitelli LJ, Gordon SE, Mello R, Frykman PN, Koziris LP, and Triplett NT(1993). Changes in Hormone Concentrations After Differentes Heavy Resistence Exercise Protocols. Journal Appl. Physiol., 75(2), 594-604.

35. Kraemer WJ, Hakkinen K, Newton RU, Nindl BN, Volek JS, McCormick M, Gotshalk L, Gordon SE, Fleck SJ, Campbell WW, Putukian M and Evans WJ (1999) Effects of heavy resistance training on hormonal response patterns in younger vs. older men. Journal Appl. Physiol. 87(3): 982-992.

36. Loebel CC, end Kraemer WJ (1998). A brief review: Testosterone and resistance exercise in men. Journal of Strength and Conditioning Research,12(1), 57-63.

37. McArdle WD, Katch FI, Katch VL (1998). Fisiologia do exercício: energia, nutrição e desempenho humano. 4 a edição. Rio de Janeiro: editora Guanabara Koogan.
38. McCall GE, Byrnes WC, Fleck SJ, Dickison A and Kraemer WJ (1999). Acute and Chronic Hormanal Responses to Resistence Training Designed to Promote Muscle Hypertrophy. Can. J. Appl. Physiol. 24 (1): 96-107.

39. MacKelvie KJ, Taunton JE, McKay HA, Khan KM (2000). Bone Mineral Density and Serum Testosterone in Chonically Trained, High Mileage 40-55 Yeas Old Male Runners. 34:273-278.

40. Marsit JL, Conley SM, Stone HM, Fleck SJ, Kearney TJ, Schirmer PG, Keit RL, Kraemer WJ, and Johnson LR (1998). Effect of ascorbic acid on serum cortisol and the testosterone cortisol ratio in junior elite weightlifters. J. Strength and Cond. Res. 12(3) 179-184.

41. Marx JO, Ratmess NA, Nindl BC, Gotshalk LA, Volek JS, Dohil K, Bush JA, Gómez AL, Mazzetti SA, Fleck SJ, Hakkinen K, Newton RU \& Kraemer WJ (2001). Low-volume circuit versus high-volume periodized resistance training in women. Medicine and Science in Sports and Exercise. V.33, n.4, pp. 635-643.

42. Nindl BC, Kraemer WJ, Daver DR, Peters JL, Marx JO, Hackman JT, Loomis GA (2001). LH secretion and testosterone concentrations are blunted after resistence exercise in men. J Appl Physiol. 91: :1251-1258 b.

43. Nindl BC, Kraemer WJ, Gotshalk LA, Marx JO, Volek JS, Bush JÁ, Hakkinen K, Newton RU, Feck SJ (2001). Testosterone responses after resistence exercise in women: Influence of regional fat distribution. International Journal of Sport Nutition and Exercise Metabolism, 11,451-465 a.

44. Ostrowski KJ, Wilson GJ, Weatherby R, Murphy PW, Lytlle AD (1997). The Effect of Weight Training Volume on Hormonal Output 
and Muscular Size and Function. Journal Strength Cond. Res. 11(3): :148-154.

45. Pagnani A, Oliveira O, Santoja R (2002). Associação Brasileira de Estudo e Combate do Doping. Manual Prático de Controle Antidoping e Alternativa Natural. $2^{\text {a }}$ edição.

46. Pullinen T, A Mero A, Huttunen P, Pakarinen A, and Komi PV (2002). Resistance exercise-induced hormonal responses in men, women, and pubescent boys. Med. Sci. Sports Exerc. Vol. 34, No. 5, pp. 806-813 a. 47. Rudolph DL and McAuley E (1998). Cortisol and Affective Responses to Exercise. Journal of Sports Sciences, 16,121-128.

48. Ryan AS,Treuth MS, Rubin MA, Miller JP, Nicklas BJ, Landis DM, Pratley RE, Libanati CR Gundberg CM and Hurley BF (1994). Effects of strength training on bone mineral density: hormonal and bone turnover relationships. J. Appl. Physiol. 77(4), 1678-1684.
49. Silva PRP, Danielski R, Czepielewski MA (2002). Esteróides anabolizantes no esporte. Revista Brasileira de Medicina do Esporte. V8, No6, 235-243.

50. Smilios I, Pilianidis T, Karamouzis M, and Tokmakidis SP (2003). Hormonal Responses after Various Resistance Exercise Protocols. Med. Sci. Sports Exerc. Vol. 35, No. 4, pp. 644-654.

51. Souissi N, Gauthier A, Sesboüe B, Larue J and Davenne D (2002). Effect of Regular Training at the Same Time of Day on Diurnal Fluctuations in Muscular Performance. Journal of Sports Sciences, 20, 929-937.

52. Tsolakis C, Messinis D, Stergioulas A, and Dessypris A (2000). Hormonal responses after strength training and detraining in prepubertal and pubertal boys. J. Strength Cond. Res. 14 (4): 399-404.

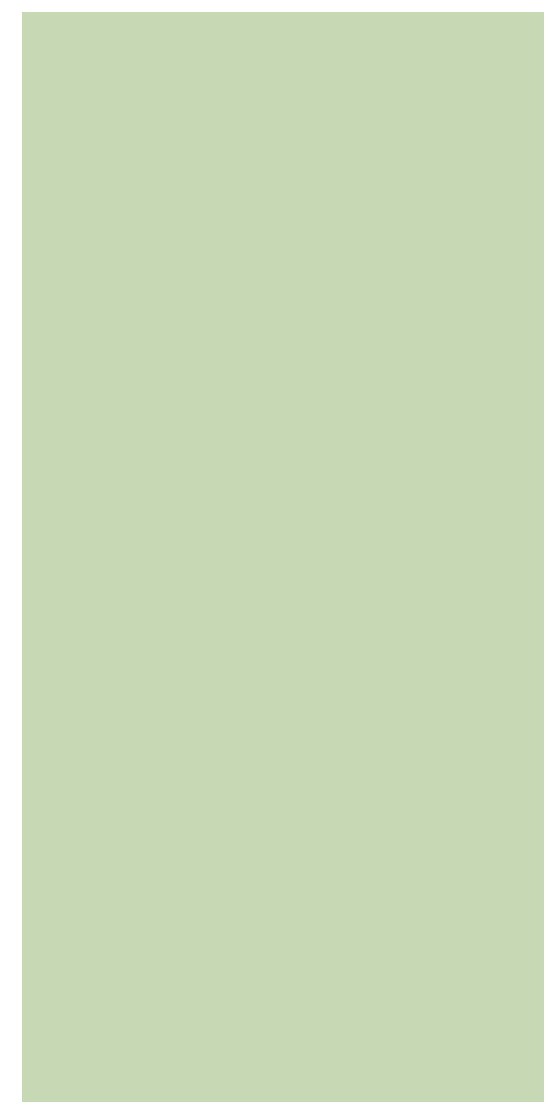

